\title{
EXTENSIONS RELATIVE TO A SERRE CLASS
}

\author{
by S. CORMACK \\ (Received 26th April 1974)
}

Consider a class $C$ of projective $R$-modules, where $R$ is a commutative ring with identity, which satisfies the conditions of (2), namely that $C$ is closed under the operations of direct sum and isomorphism and $C$ contains the zero module. Following (2) a module $M$ is said to have $C$-cotype $n$ (respectively $C$-type $n$ ) if it has a projective resolution $\ldots \rightarrow P_{n} \rightarrow \ldots \rightarrow P_{0} \rightarrow M \rightarrow 0$ with $P_{i} \in C$ for $i>n$ (respectively $P_{i} \in C$ for $i \leqq n$ ). Let $S$ be the class of modules of $C$-cotype -1 , equivalently of $C$-type infinity. It is assumed throughout that $S$ is a Serre Class. We define an abelian category $\mathscr{S}$ of modules with the property that $C$-cotype is homological dimension in $\mathscr{S}$, while in the case $C=0, S$ is just the category of $\boldsymbol{R}$-modules. It follows that all categorical results on homological dimension also hold for cotype.

In Theorem 12 the restriction to a Serre class $S$ is expressed in terms of the coherence of the ring $R$. Some examples of such classes are given.

Repeated use is made of the following result of (2).

Theorem 1. Suppose $0 \rightarrow L \rightarrow M \rightarrow N \rightarrow 0$ is an exact sequence of $R$-modules. Then for all $n \geqq-1$,

(i) if $L$ has cotype $(n-1)$ and $M$ has cotype $n$, then $N$ has cotype $n$,

(ii) if $L$ has cotype $n$ and $N$ has cotype $n$, then $M$ has cotype $n$,

(iii) if $M$ has cotype $n$ and $N$ has cotype $(n+1)$, then L has cotype $n$.

Corollary. If $0 \rightarrow L \rightarrow M \rightarrow N \rightarrow 0$ is exact and any two of $L, M, N$ belong to $S$ then so does the third. Also $0 \in S$.

It follows that $\boldsymbol{S}$ is a Serre Class if and only if it is closed under submodules; or equivalently, closed under quotient modules.

\section{Definition of $\mathscr{S}$}

The category $\mathscr{S}$ has as objects all $R$-modules. The morphisms from $A$ to $B$ are equivalence classes of $S$-homomorphisms in the sense of Serre (3). The definitions are as follows. Let $G$ be a submodule of $A \oplus B$ and let $p: G \rightarrow A, q: G \rightarrow B$ be the projections. $G$ is an $S$-homomorphism from $A$ to $B$ if $\operatorname{Ker} p$ and Coker $p$ both belong to $S$ (that is, if $p$ is an $S$-isomorphism in the sense of (3)). A relation is defined on $S$-homomorphisms from $A$ to $B$ by $G \sim H$ if and only if the inclusions $G \cap H \rightarrow G$ and $G \cap H \rightarrow H$ are $S$-isomorphisms; 
that is, if and only if $G / G \cap H$ and $H / G \cap H$ both belong to $S$. Hence $G \sim H$ if and only if $G$ and $H$ are $S$-equal.

Suppose $G \sim H$ and $H \sim K$. Now $G / G \cap K$ is an extension of a submodule of $H / H \cap K$ by a quotient of $G / G \cap H$. Since $S$ is a Serre class this implies that $G / G \cap K \in S$. This proves that $G \sim K$. We therefore define $\operatorname{Hom}_{\mathscr{S}}(A, B)$ as the set of equivalence classes of $S$-homomorphisms from $A$ to $B$. For simplicity of notation we will write $G$ for both an $S$-homomorphism and its equivalence class.

The identity of $\operatorname{Hom}_{\mathscr{S}}(A, A)$ is the diagonal subset $D$ of $A \oplus A$ with the composition of $G \in \operatorname{Hom}_{\mathscr{S}}(A, B)$ and $H \in \operatorname{Hom}_{\mathscr{S}}(B, C)$ defined by

$$
H G=\{(a, c) \in A \oplus C: \exists b \in B \text { with }(a, b) \in G \text { and }(b, c) \in H\} .
$$

Lemma 2. $H G$ is an $S$-homomorphism and the composition is well defined on equivalence classes. Thus $\mathscr{S}$ is a category.

Proof. If

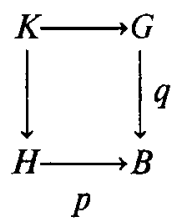

is a pullback square, $K \rightarrow G$ is an $S$-isomorphism and hence so is the composition $K \rightarrow A$ in the following commutative square.

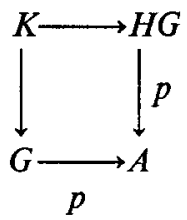

Here, $K \rightarrow H G$ is the canonical epimorphism. Hence $p: H G \rightarrow A$ is an $S$ isomorphism.

Now suppose $G \sim G^{\prime}$ and $K, K^{\prime}$ are the corresponding pullbacks. Then $K / K \cap K^{\prime} \rightarrow G / G \cap G^{\prime}$ is a monomorphism and there is an epimorphism from $K / K \cap K^{\prime}$ to $H G / H G \cap H G^{\prime}$. Thus $H G / H G \cap H G^{\prime} \in S$ and $H G \sim H G^{\prime}$.

Similar techniques may be used to prove that $\mathscr{S}$ is an abelian category with the following definitions, where $G, H \in \operatorname{Hom}_{\mathscr{S}}(A, B)$.

(i) Let $G+H=\left\{\left(a, b_{1}+b_{2}\right):\left(a, b_{1}\right) \in G\right.$ and $\left.\left(a, b_{2}\right) \in H\right\}$. Then $\operatorname{Hom}_{\mathscr{S}}(A, B)$ is an abelian group with zero $N=\{(a, 0): a \in A\}$ and inverse $-G=\{(a, b):(a,-b) \in G\}$.

(ii) Let $X=p(G \cap N) . \quad X$ is the submodule of elements $a$ in $A$ such that $(a, 0) \in G$. Let $i: X \rightarrow A$ be the inclusion and $K=\{(x, i(x)): x \in X\}$. Then $K \in \operatorname{Hom}_{\mathscr{S}}(X, A)$ is a kernel of $G$.

(iii) Let $Y=B / q(G)$ and let $j: B \rightarrow Y$ be the projection. Define $C=\{(b, j(b)): b \in B\}$. Then $C \in \operatorname{Hom}_{\mathscr{P}}(B, Y)$ is a cokernel of $G$. 
(iv) Let $Z=q(G)$ with inclusion $k: Z \rightarrow B$. Define $I=\{(a, z):(a, k(z)) \in G\}$ and $J=\{(z, k(z)): z \in Z\}$. Then $I \in \operatorname{Hom}_{\mathscr{S}}(A, Z)$ is an image of $G$ and $J \in \operatorname{Hom}_{\mathscr{S}}(Z, B)$ is a coimage of $G$.

We therefore have the following analysis of $G$.

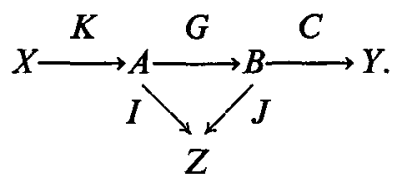

Theorem 3. $G \in \operatorname{Hom}_{\mathscr{S}}(A, B)$ is a monomorphism (epimorphism) in $\mathscr{P}$ if and only if $q: G \rightarrow B$ is an $S$-monomorphism (epimorphism).

Proof. $G$ is a monomorphism in $\mathscr{S}$ if and only if $K \sim N$ as submodules of $X \oplus A$. But $K \cap N=0$ so $K \sim N$ if and only if $K \in S$ and $N \in S . K$ and $N$ are both isomorphic to $X$ which is isomorphic to $\operatorname{Ker}(q: G \rightarrow B)$. Hence $K \in S$ and $N \in S$ if and only if $\operatorname{Ker} q \in S$. The dual result is proved similarly.

It follows that $A$ and $B$ are $S$-isomorphic in the sense of Serre if and only if there is an isomorphism $G \in \operatorname{Hom}_{\mathscr{S}}(A, B)$. The null objects of the category $\mathscr{S}$ are the modules in $S$ and we have

Proposition 4. $\operatorname{Hom}_{\mathscr{S}}(A, B)=0$ for all $B$ if and only if $A \in S$.

Proof. If $\operatorname{Hom}_{\mathscr{S}}(A, A)=0$, then $D \sim N$; but $D \cap N=0$ and $A$ is isomorphic to $N$. Therefore $A \in S$. Conversely if $A \in S$ and $G \in \operatorname{Hom}_{\mathscr{S}}(A, B)$ then $p: G \rightarrow A$ is an $S$-isomorphism so $G \in S$. It follows that $G \sim N$.

Finally note that if $S=0$ then $\mathscr{S}$ reduces to the category of $R$-modules and module homomorphisms.

\section{Extensions in $\mathscr{S}$}

The functors $\mathrm{Ext}^{n}$ can be defined in any abelian category which has sufficient projectives. We show that every projective $R$-module is a projective object of $\mathscr{S}$. Hence every $R$-module has a projective resolution in $\mathscr{S}$.

Lemma 5. If $A$ and $B$ are $S$-isomorphic then they have the same cotype.

Proof. It is sufficient to prove that if $f: A \rightarrow B$ is an $S$-isomorphism then $A$ and $B$ have the same cotype. But now let

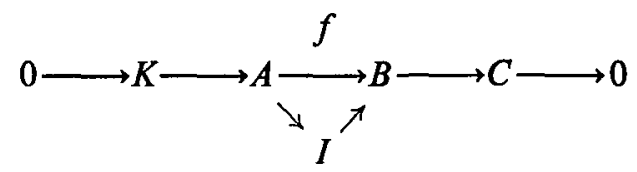

be exact with $K, C \in S$. The result follows immediately from Theorem 1 . 
Theorem 6. A module $A$ of $C$-cotype zero is a projective object in $\mathscr{S}$.

Proof. Suppose $G \in \operatorname{Hom}_{\mathscr{O}}(A, C)$ and let $H \in \operatorname{Hom}_{\mathscr{C}}(B, C)$ be an epimorphism is $\mathscr{S}$. We must produce $K \in \operatorname{Hom}_{\mathscr{S}}(A, B)$ such that $H K \sim G$. The first step reduces the problem to the case in which $q: H \rightarrow C$ is epi.

Let $X=q(H)$ and $i: X \rightarrow C$ the inclusion. Then $i$ is an $S$-isomorphism since $H$ is an epimorphism in $\mathscr{S}$ and $q: H \rightarrow X$ is epi. Also $H$ can be considered as $H^{\prime} \in \operatorname{Hom}_{\mathscr{P}}(B, X)$. Let $G^{\prime}=\{(a, x) \in A \oplus X:(a, i(x)) \in G\}$. Then $G^{\prime} \rightarrow G$ is mono and so is the map $G / G^{\prime} \rightarrow C / i(X)$ induced by the projections. Hence $G^{\prime} \rightarrow G$ is an $S$-isomorphism; therefore so is the composition $G^{\prime} \rightarrow G \rightarrow A$. Thus $G^{\prime} \in \operatorname{Hom}_{\mathscr{S}}(A, X)$ while $H^{\prime} \in \operatorname{Hom}_{\mathscr{S}}(B, X)$ is such that $q: H^{\prime} \rightarrow X$ is epi. Moreover, if $K \in \operatorname{Hom}_{\mathscr{P}}(A, B)$ satisfies $H^{\prime} K \sim G^{\prime}$, then $H K=i H^{\prime} K \sim i G^{\prime}=G$. It is therefore sufficient to prove the result in the case where $q: H \rightarrow C$ is epi.

Now by Lemma $5, G$ has cotype 0 since $A$ does; so by definition there is an exact sequence $0 \rightarrow X \rightarrow P \stackrel{\Phi}{\rightarrow} G \rightarrow 0$ with $P$ projective and $X \in S$. Hence there exists a map $\alpha: P \rightarrow H$ such that the following diagram is commutative.

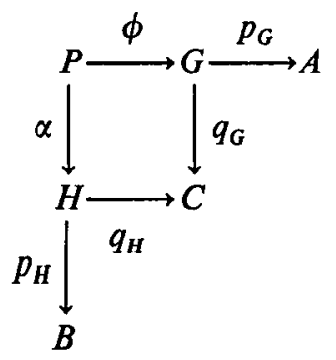

For clarity the various projection maps are here distinguished by subscripts. Let $\pi=p_{G} \phi, \theta=p_{H^{\alpha}}$ and $\beta=q_{G} \phi=q_{H} \alpha$. Now define $K \subseteq A \oplus B$ as $K=(\pi, \theta)(P)$. The diagram

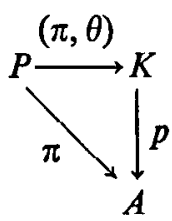

is commutative with $\pi$ an $S$-isomorphism and $(\pi, \theta)$ ep;. Therefore $K \in \operatorname{Hom}_{\mathscr{S}}(A, B)$.

It remains to verify that $H K \sim G$. If $(a, c) \in G$, choose $x \in P$ with $\phi(x)=(a, c)$. Then $\alpha(x)=(\theta(x), c) \in H$ and $\pi(x)=a$, so $(a, \theta(x)) \in K$. Hence $(a, c) \in H K$; that is $G \subseteq H K$. Let $Y=\operatorname{Ker} p_{H}$ which belongs to $S$. Then $q=q_{H} \mid Y: Y \rightarrow C$ is mono so $q(Y) \in S$. Hence the quotiert $Z$ of $q(Y)$ modulo the subgroup $q(Y) \cap q_{G}\left(\operatorname{Ker} p_{G}\right)$ also belongs to $S$. We define a map $\psi: H K \rightarrow Z$ with kernel $G$. This will complete the proof by showing that $H K / G$ is isomorphic to a subgroup of $Z$ and hence belongs to $S$.

To define $\psi$ let $(a, c) \in H K$. Choose $x \in P$ such that $\pi(x)=a$ and $(\theta(x), c) \in H$. Let $z=c-\beta(x)$. Since $(0, z)=(\theta(x), c)-\alpha(x)$, which belongs 
to $H$, we have $z \in q(Y)$. Define $\psi(a, c)$ as the equivalence class represented by $z$. If $x^{\prime}$ is another choice, then

$$
z-z^{\prime}=\beta\left(x^{\prime}\right)-\beta(x) \text { and }\left(0, \beta\left(x^{\prime}\right)-\beta(x)\right)=\phi\left(x^{\prime}\right)-\phi(x) \in \operatorname{Ker} p_{G} .
$$

Hence $z$ and $z^{\prime}$ represent the same element of $Z$. Finally,

$$
(a, c)=\phi(p)+(0, c-\beta(p))
$$

so $(a, c) \in \operatorname{Ker} \psi$ if and only if $(0, c-\beta(p)) \in G$. It follows that $\operatorname{Ker} \psi=G$. This completes the proof.

Corollary 7. $\mathscr{S}$ has sufficient projectives.

Proof. Every projective module has cotype zero and so is a projective object in $\mathscr{S}$. Hence a projective resolution in the module category gives rise to a projective resolution in $\mathscr{S}$.

The functors $\operatorname{Ext}_{\mathscr{S}}^{n}$ are therefore defined and $\operatorname{Ext}_{\mathscr{S}}^{1}(A, B)=0$ for all $B$ if and only if $A$ is a projective object in $\mathscr{S}$.

Lemma 8. If $A$ has finite cotype and $\operatorname{Ext}_{\mathscr{S}}^{1}(A, B)=0$ for all $B$ then $A$ has cotype 0 .

Proof. Suppose $A$ has cotype $r$. There is an exact sequence

$$
0 \rightarrow K \rightarrow P \rightarrow A \rightarrow 0
$$

with $P$ projective and $K$ of cotype $(r-1) . \quad A$ is a projective object of $\mathscr{S}$; so this sequence splits in $\mathscr{S}$ giving an isomorphism $G \in \operatorname{Hom}_{\mathscr{S}}(A \oplus K, P)$. Hence $A \oplus K$ has cotype 0 . Theorem 1 (iii) applied to the sequence

$$
0 \rightarrow A \rightarrow A \oplus K \rightarrow K \rightarrow 0
$$

shows that (if $r \geqq 2$ ) $A$ has cotype $(r-2)$. Hence $A$ has cotype 0 .

Theorem 9. If $A$ has finite cotype, then $\operatorname{Ext}_{\mathscr{S}}^{n+1}(A, B)=0$ for all $B$ if and only if $A$ has cotype $n(n \geqq-1)$.

Proof. Proposition 4 gives the result for $n=-1$. Theorem 6 and Lemma 8 prove the result for $n=0$. We use induction on $n>0$. Suppose $A$ has cotype $r$. The exact sequence $\left(^{*}\right)$ gives rise to a long exact sequence

$$
\ldots \quad \rightarrow \operatorname{Ext}_{\mathscr{S}}^{n}(P, B) \rightarrow \operatorname{Ext}_{\mathscr{S}}^{n}(K, B) \rightarrow \operatorname{Ext}_{\mathscr{S}}^{n+1}(A, B) \rightarrow \operatorname{Ext}_{\mathscr{S}}^{n+1}(P, B) \rightarrow \ldots
$$

for all $B$. $\operatorname{Ext}_{\mathscr{S}}^{n}(P, B)=0$ by Theorem 6 and $\operatorname{Ext}_{\mathscr{S}}^{n+1}(A, B)=0$ by hypothesis. Hence $\operatorname{Ext}_{\mathscr{S}}^{n}(K, B)=0$; so by induction, $K$ has cotype $(n-1)$. It follows that $A$ has cotype $n$. Conversely, if $A$ has cotype $n(n \geqq 0)$, then $K$ has cotype $(n-1)$ so $\operatorname{Ext}_{\mathscr{S}}^{n}(K, B)=0=\operatorname{Ext}_{\mathscr{S}}^{n+1}(P, B)$ which implies the result.

This theorem shows that, as long as modules have finite cotype, then cotype is simply homological dimension in the category $\mathscr{S}$. We also see that the $C$-cotype of a module depends only on the derived class $S$. Note that $C \subseteq S$; Since $C \in C$ it has a projective resolution $0 \rightarrow C \rightarrow C \rightarrow 0$. $C$ is determined by $S$ 
provided $\boldsymbol{C}$ is closed under direct summands, since then $\boldsymbol{C}=\boldsymbol{S} \cap \boldsymbol{P}$ where $\boldsymbol{P}$ is the class of projective modules (2, Theorem 2). In general the following relations hold between $\boldsymbol{C}$ and $\boldsymbol{S}$.

Lemma 10. If $C$ and $C^{\prime}$ both have derived class $S$ then for every $C \in C$ there exists $X \in S \cap P$ and $C^{\prime} \in C^{\prime}$ such that $C^{\prime}=C \oplus X$.

To prove this, note that if $C \in C \subseteq S$ there is an exact sequence $0 \rightarrow X \rightarrow C^{\prime} \rightarrow C \rightarrow 0$ with $X \in S$ and $C^{\prime} \in C^{\prime}$. The result follows since $C$ is projective.

In particular, for a given class $C$ with derived class $S$, let $C^{\prime}=S \cap P$. This is a class. Let $S^{\prime}$ be its derived class. $C \subseteq C^{\prime}$ so $S \subseteq S^{\prime}$. Conversely if $A \in S^{\prime}$ there is an exact sequence

$$
\ldots \rightarrow C_{n}^{\prime} \rightarrow \ldots \rightarrow C_{0}^{\prime} \rightarrow A \rightarrow 0
$$

with $C_{i}^{\prime} \in S \cap P$ for all $i$. Thus if either $S$ is Serre or the sequence is finite we find that $A \in S$ (by the Corollary to Theorem 1). Hence, for a Serre class $S, S \cap P$ also has derived class $S$.

Corollary 11. $C \subseteq S \cap P$ with equality if and only if $C$ is closed under direct summands. If $\boldsymbol{S}$ is Serre, then for every $C^{\prime} \in S \cap P$ there exists $X \in S \cap P$ such that $C^{\prime} \oplus X \in C$.

\section{Conditions for $\mathbf{S}$ to be a Serre class}

The ring $R$ is said to be $(0, C)$-coherent if every module of $C$-type 0 belongs to $S$.

Theorem 12. $S$ is a Serre class if and only if $R$ is $(0, C)$-coherent.

Proof. Suppose $S$ is Serre and $A$ has $C$-type 0 . Then $A$ has a projective resolution $\ldots \rightarrow P \rightarrow C \rightarrow A \rightarrow 0$ with $C \in C \subseteq S$. Hence $A$, being a quotient of $C$, belongs to $S$. Conversely, let $A \in S$ and $A \rightarrow B$ epi. $A$ has type 0 , therefore by (1, Lemma 7) $B$ has type 0 and so belongs to $S$. Hence $S$ is closed under quotients.

The following are examples of classes for which $S$ is Serre. For proofs see (1).

(i) The class $\boldsymbol{F}$ of finitely generated free modules over a Noetherian ring $R$. Then $S$ is the class of finitely generated $R$-modules.

(iii) The class $D$ of free graded $R$-modules with generators in only a finite number of dimensions, where $R$ is a finite dimensional ring. Then $S$ is the class of $R$-modules with generators in only a finite number of dimensions.

\section{REFERENCES}

(1) J. F. Adams, Lectures on generalised cohomology, lecture 5. Lecture notes vol. 99 (Springer-Verlag, Berlin, 1969). 
(2) S. Cormack, An analogue of homological dimension using a general class of projective modules, J. London Math. Soc. (2) 1 (1969), 760-764.

(3) J. P. Serre, Groupes d'homotopie et classes de groupes abéliens, Ann. of Math. 58 (1953), 258-294.

UNIVERSITY OF EDINBURGH 